\title{
Trial Summary Parameter Version of the Reference Terminology
}

National Cancer Institute

\section{Source}

National Cancer Institute. Trial Summary Parameter Version of the Reference

Terminology. NCl Thesaurus. Code C117461.

The version information of the terminology system that is being used for the trial summary parameter. 